\title{
Correction to: CircPAN3 ameliorates myocardial ischaemia/ reperfusion injury by targeting miR-421/Pink1 axis-mediated autophagy suppression
}

\author{
Cheng-Long Zhang · Tian-Yi Long · Si-Si Bi · Sayed-Ali Sheikh · Fei Li
}

Published online: 22 January 2021

(c) The Author(s), under exclusive licence to United States and Canadian Academy of Pathology 2021

Correction to: Laboratory Investigation

https://doi.org/10.1038/s41374-020-00483-4

The original version of this article unfortunately contained a mistake in Fig. 6. The data of Fig. 6b showed the relation expression of "miR-421", while the authors mistakenly wrote "pink1" in the y-axis. As for Fig. 6c, the authors missed the data of pink1 mRNA level by qRT-PCR assay. The authors apologize for the mistake. The original article has been corrected. 
A

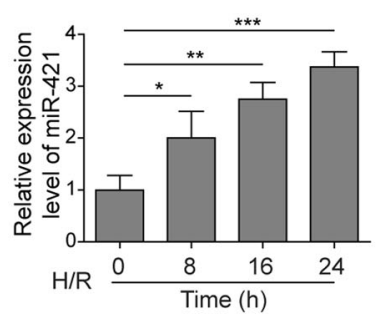

D

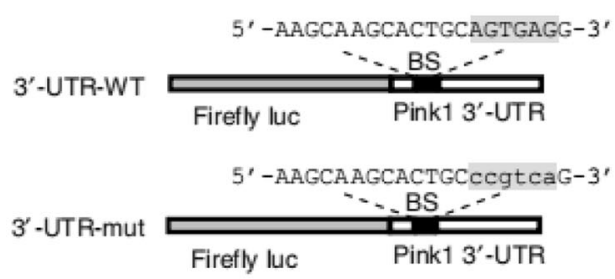

F

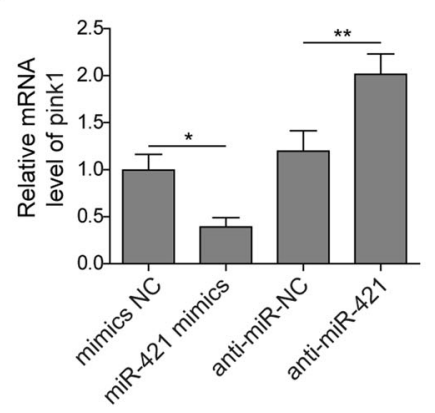

B

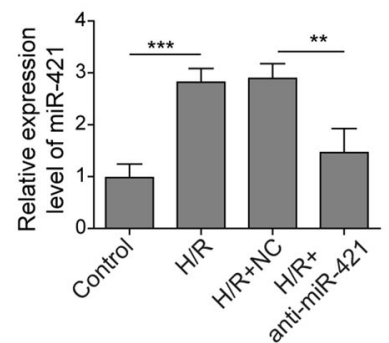

G

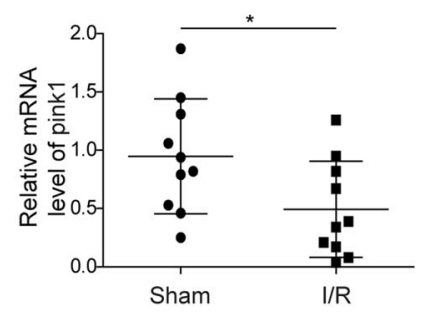

C

$E$
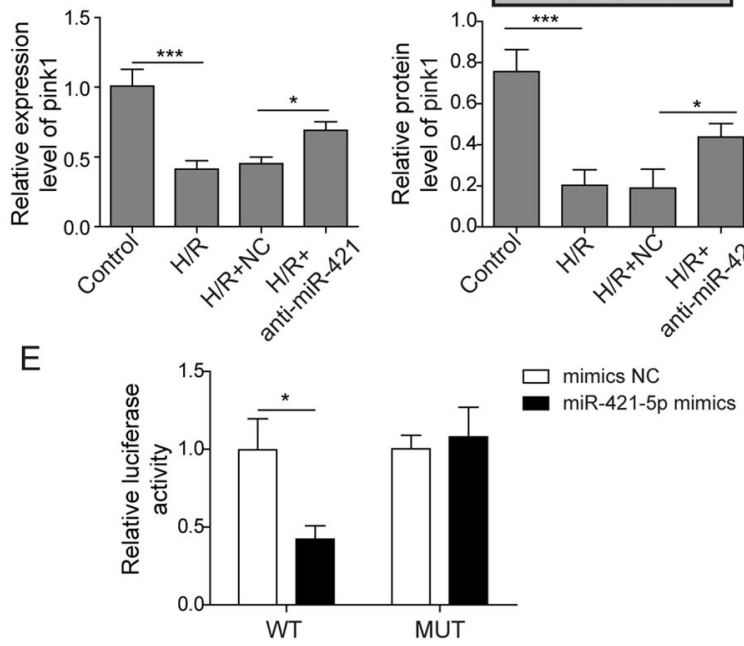

$\mathrm{H}$

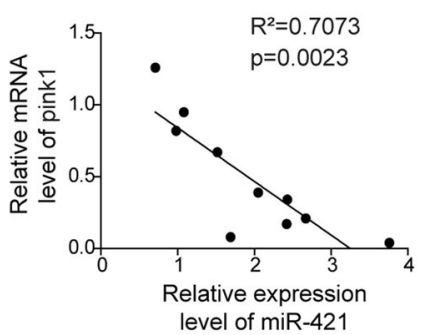

Fig. 6. 